\title{
ON METABELIAN GROUPS
}

\author{
BY
}

\section{WILLIAM BENJAMIN FITE}

\section{Introduction.}

The concept of an isomorphism between two groups was introduced by Jordan in 1868. In the Comptes Rendus of that year, vol. 66, p. 836, he defined an $\alpha, 1$ isomorphism. Ten years later this concept was generalized to that of an $\alpha, \beta$ isomorphism by CAPELLI in the Giornali di Matema tiche, vol. 16, 1878, p. 33.

Every simple isomorphism of a group with itself may be looked upon as a substitution that replaces each operator of the group by the operator that corresponds to it in this isomorphism. It was first observed by HöLDER $\dagger$ and MOone $\ddagger$ that the totality of these substitutions forms a group. This group is called the group of isomorphisms of the given group.

An isomorphism of a group with itself produced by making every operator of $G$ correspond to its transform with respect to some operator of $G$ is called a cogredient isomorphism. To the totality of cogredient isomorphisms of $G$ corresponds an invariant subgroup of the group of isomorphisms of $G$. This subgroup is called the group of cogredient isomorphisms of $G . \S$

We define a Metabelian Group as a group whose group of cogredient isomorphisms is abelian.

The group of cogredient isomorphisms of a group $G$ is simply isomorphic with the quotient group of $G$ with respect to the subgroup formed by the invariant operators of $G . \|$ If $G$ is metabelian this quotient group is abelian, and therefore the commutators $\uparrow$ of $G$ are invariant. Conversely, if the commutators of $G$ are invariant, $G$ is metabelian.** Hence we could define a metabelian group as a group whose commutators are invariant.

* Presented to the Society August 25, December 28, 1899, and February 23, 1901, under various titles. Received for publication February 3, 1902.

† HöLDmr, Mathematische Annalen, vol. 43 (1893), p. 314.

$\ddagger$ Moore, Bulletin of the American Mathematical Society, vol. 1 (1894), p. 61.

\& HöldDER, loc. cit., p. 314.

|| This is given implicitly by HöLDER, loc. oit., pp. 329, 330.

TDeDEKIND, Mathematische Annalen, vol. 48 (1897), p. 553.

** Miller, Bulletin of the American Mathe matical Society, vol. 4 (1898), pp. 137, 135 . 
It follows from the definition that every subgroup (and likewise every quotient group) of a metabelian group is either metabelian or abelian.

Let $G^{\prime}$ be the group of cogredient isomorphisms of a group $G$, and $G^{\prime \prime}$ that of $G^{\prime}$, and so on. $\dagger$ Then if the series $G, G^{\prime}, G^{\prime \prime}, \ldots$ ends with identity, $G$ is the direct product of groups of orders $p_{1}^{a_{1}}, p_{2}^{a_{2}}, \cdots, p_{n}^{a_{n}}$ respectively, where $p_{1}^{a_{1}} p_{2}^{a_{2}} \cdots p_{n}^{a_{n}}$ is the order of $G$ and $p_{1}, p_{2}, \cdots, p_{n}$ are distinct primes $f$. Conversely, if $G$ is the direct product of groups of orders $p_{1}^{a_{1}}, p_{2}^{a_{2}}, \ldots, p_{n}^{a_{n}}$ respectively, it is evident that we shall arrive at identity by forming these successive groups of cogredient isomorphisms. $§$ In particular, a metabelian group of order $p_{1}^{a_{1}} p_{2}^{a_{2}} \cdots p_{n}^{a_{n}}$ is the direct product of groups of orders $p_{1}^{a_{1}}, p_{2}^{a_{2}}, \cdots, p_{n}^{a_{n}}$ respectively.

We shall designate by $G^{\prime}$ the group of cogredient isomorphisms of the group $G$. Whenever we speak of the operators of $G$ corresponding to the operators of $G^{\prime}$ we shall suppose that $G$ and $G^{\prime}$ are arranged in an $\alpha, 1$ isomorphism, the $\alpha$ invariant operators of $G$ corresponding to identity of $G^{\prime}$.

A brief summary of the different sections follows.

In section 1 it is shown that certain abelian groups cannot be groups of cogredient isomorphisms. In addition, some limitations on the order of the group of cogredient isomorphisms under certain conditions are given, together with some theorems on the order of the operators of the group of cogredient isomorphisms. There is also a theorem on the order of an abelian subgroup that is always contained in a metabelian group of order $p^{m}$, where $p$ is a prime.

The question of the order of the product of two operators of a metabelian group is considered in section 2.

In the second edition of his Algebra, vol. 2, p. 133, Weber states, without proof, that the product of two commutators is not necessarily a commutator. It is the object of section 3 to show that there are certain metabelian groups whose commutator subgroups contain operators that are not commutators.

The number of metabelian groups whose order is a given power of a prime and whose invariant operators form cyclic groups is determined in section 4 . It is shown that this number depends only on the different orders of the independent generators of the groups of cogredient isomorphisms and is independent of the number of these generators.

In section 5 a similar, but somewhat more limited, investigation is made concerning metabelian groups whose order is a given power of a prime and whose invariant operators form a subgroup that is the direct product of two cyclic groups of unequal orders, the commutator subgroup being contained in that one of these cyclic subgroups which is of the greater order.

†Cf. Ahrens, Leipziger Berich te, Mathematische-Physiche Klasse, vol. 49 (1897), pp. $616-626$.

$\ddagger$ BURnside, Theory of Groups of Finite Order, 1897, p. 115.

§ LoEwy, Mathematische Annalen, vol. 55 (1901), pp. 68, 69. 
Section 6 contains a discussion of groups that have metabelian groups of cogredient isomorphisms. It is shown that there are certain metabelian groups that cannot be groups of cogredient isomorphisms. Some of the theorems of this section are similar to those of section 1. An application of the results of this section is made to groups of orders $p^{5}$ and $p^{6}$, where $p$ is a prime.

I am indebted to Professor Miller for helpful suggestions and criticisms in the preparation of this paper.

\section{$\S 1$. Abelian groups of cogredient isomorphisms.}

It is known that $G^{\prime}$ cannot be cyclic nor the direct product of two cyclic groups of different orders, and that if it is abelian it has no operator of order greater than the order of the subgroup formed by the invariant operators of $G$.*

Let $H$ denote the subgroup formed by the invariant operators of $G$. Suppose that $G^{\prime}$ is abelian with the independent generators $A_{1}^{\prime}, A_{2}^{\prime}, \ldots$ of orders $a_{1}^{\prime}, a_{2}^{\prime}, \ldots$ respectively. Let $A_{1}, A_{2}, \ldots$ be operators of $G$ that correspond respectively to $A_{1}^{\prime}, A_{2}^{\prime}, \ldots$. Since $G^{\prime}$ is abelian, we have

$$
A_{j}^{-1} A_{1} A_{j}=h_{j} A_{1} \quad(j=2,3, \cdots),
$$

where the $h_{j}$ are operators of $H$. From this we get

$$
A_{i}^{-1} A_{1^{j}}^{a_{j}^{\prime}} A_{j}=h_{j}^{a_{j}^{\prime}} A_{1^{j}}^{a_{j}^{\prime}}=A_{1^{j}}^{a_{j}^{\prime}} .
$$

If now $G$ is of order $p^{m}$, where $p$ is a prime, and we denote by $a_{n}^{\prime}$ the greatest of the orders $a_{2}^{\prime}, a_{3}^{\prime}, \ldots$, then $A_{1^{a_{n}^{\prime}}}^{i^{2}}$ commutative with every operator of $G$ and is accordingly contained in $H$. But.this is possible only if $a_{1}^{\prime}$ is equal to, or less than, $a_{n}^{\prime}$. Therefore if $G$ is metabelian of order $p^{m}, G^{\prime}$ must have at least two independent generators of the highest order.

Now a metabelian group $G$ of order $p_{1}^{a_{1}} p_{2}^{a_{2}} \cdots p_{n}^{a_{n}}$, where $p_{1}, p_{2}, \cdots, p_{n}$ are distinct primes, is the direct product of groups of orders $p_{1}^{a_{1}}, p_{2}^{a_{2}}, \cdots, p_{n}^{a_{n}}$ respectively (see introduction), and $G^{\prime}$ is the direct product of the groups of cogredient isomorphisms of the respective factor groups. $\dagger$ Let $A_{1}^{\prime}, A_{2}^{\prime}, \ldots, A_{n}^{\prime}$ be operators of the highest order in the groups of cogredient isomorphisms of the respective factor groups. Let these highest orders be $p_{1}^{a_{1}^{\prime}}, p_{2}^{a_{2}^{\prime}}, \ldots, p_{n^{n}}^{a_{n}^{\prime}}$ respectively. From what has just been proved it follows that there are operators $B_{1}^{\prime}, B_{2}^{\prime}, \ldots, B_{n}^{\prime}$ in these groups of cogredient isomorphisms of orders

$$
p_{1}^{a_{1}^{\prime}}, p_{2}^{a_{2}^{\prime}}, \cdots, p_{n}^{a_{n}^{\prime}}
$$

respectively that are independent of $A_{1}^{\prime}, A_{2}^{\prime}, \ldots, A_{n}^{\prime}$. Therefore

$$
A_{1}^{\prime} A_{2}^{\prime} \cdots A_{n}^{\prime} \quad \text { and } B_{1}^{\prime} B_{2}^{\prime} \cdots B_{n}^{\prime}
$$

* Miller, Comptes Rendas de l'A cadémie des Sciences, vol. 128 (1899), p. 229.

$\dagger$ Mrller, Bulletin of the American Mathematical Society, vol. 5 (1899), p. 294. 
are two independent generators of $G^{\prime}$ of the highest order $p_{1}^{a_{1}^{\prime}} p_{2}^{a_{2}^{\prime}} \cdots p_{n}^{a_{n}^{\prime}}$, and the order of every other independent generator of $G^{\prime}$ is a divisor of $p_{1}^{a_{1}^{\prime}} p_{2}^{a_{2}^{\prime}} \ldots p_{n}^{a_{n}^{\prime}}$. We have proved therefore

THEOREM I.-The group of cogredient isomorphisms of a group $G$ cannot be the direct product of cyclic groups whose orders are such that any one of them is not a divisor of at least one of the others.

Now $a_{1}^{\prime}$ is the least common multiple of the orders of $h_{2}, h_{3}, \ldots$ But these operators (the commutators formed by $A_{1}$ and all the operators of $G$ ) form an abelian group, and in any abelian group the least common multiple of the orders of any operators is the order of some operator.* Therefore $a_{1}^{\prime}$ is the order of a commutator of $G$. This result can be stated as follows :

TheOREM II.-The order of every operator of the group of cogredient isomorphisms of a metabelian group $G$ is the order of a commutator of $G$.

If $G^{\prime}$ is abelian the order of $H$ is divisible by every prime factor of the order of $G$, and therefore if the order of $G$ were not divisible by the cube of a prime, $G^{\prime}$ would be cyclic. Hence, no group whose order is not divisible by the cube of a prime can be metabelian.

The least common multiple of the orders of any operators of a group $G$ that is the direct product of groups whose orders are respectively powers of distinct primes is the order of some operator of $G . \dagger$ In particular, this is true of metabelian groups.

Theorem III.-If $G$ is a metabelian group of order $g$ and contains an abelian subgroup of order $g / p_{1}^{a_{1}} p_{2}^{a_{2}} \cdots p_{n}^{\alpha_{n}}$, where $p_{1}, p_{2}, \cdots, p_{n}$ are distinct primes, the order of every operator of $G^{\prime}$ is a divisor of $p_{1}^{a_{1}} p_{2}^{a_{2}} \cdots p_{n}^{a_{n}}$.

For, let $G_{1}$ denote the abelian subgroup of $G$ of order $g / p_{1}^{a_{1}} p_{2}^{a_{2}} \cdots p_{n}^{a_{n}}$ and $G_{1}^{\prime}$ the subgroup of $G^{\prime}$ that corresponds to $G_{1}$. It may be assumed that $G_{1}$ contains $H$. If $A_{1}$ is any operator of $G_{1}$ that is not contained in $H$, it is commutative with at least $g / p_{1}^{\alpha_{1}} p_{2}^{\alpha_{2}} \cdots p_{n}^{a_{n}}$ operators of $G$ and therefore the number of its conjugates in $G$ is a divisor of $p_{1}^{a_{1}} p_{2}^{a_{2}} \cdots p_{n}^{a_{n}}$ : The number of commuta. tors of $G$ formed. by $A_{1}$ and all the operators of $G$ is therefore a divisor of $p_{1}^{a_{1}} p_{2}^{a_{2}} \cdots p_{n}^{a_{n}}$. These commutators form a group. Therefore the order of the operator of $G_{1}^{\prime}$ that corresponds to $A_{1}$, that is, the order of any operator of $G_{1}^{\prime}$, is a divisor of $p_{1}^{a_{1}} p_{2}^{a_{2}} \cdots p_{n}^{a_{n}}$.

If $A_{2}$ is any operator of $G$ that is not contained in $G_{1}$, then some power of $A_{2}$ of the form

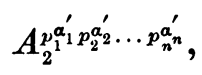

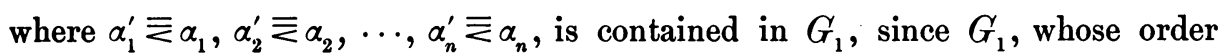
is $g / p_{1}^{a_{1}} p_{2}^{a_{2}} \cdots p_{n}^{a_{n}}$, and $A_{2}$ generate a subgroup of $G$. But if

* Frobenius and Stickelberaer, Crelle's Journal, vol. 86 (1879), p. 234.

†The proof of this is similar to that given by FrobeniUs and STICKELBERGER, loc. cit., for abelian groupş. 


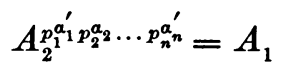

then $A_{1}$ is commutative with at least $g / p_{1}^{a_{1}-a_{1}^{\prime}} p_{2}^{a_{2}-a_{2}^{\prime}} \ldots p_{n}^{a_{n}-a_{n}^{\prime}}$ operators of $G$ and the number of its conjugates in $G$ is a divisor of $p_{1}^{a_{1}-a_{1}^{\prime}} p_{2}^{a_{2}-a_{2}^{\prime}} \ldots p_{n_{n}^{a}}^{a_{n}} a_{n}^{\prime}$. The order of the corresponding operator of $G^{\prime}$ is therefore a divisor of

$$
p_{1}^{a_{1}-a_{1}^{\prime}} p_{2}^{a_{2}-a_{2}^{\prime}} \cdots p_{n}^{a_{n}-a_{n}^{\prime}} .
$$

Hence the order of every operator of $G^{\prime}$ is a divisor of $p_{1}^{a_{1}} p_{2}^{a_{2}} \cdots p_{n}^{a_{n}}$, and the theorem is proved.

If $G_{1}$ is a maximum abelian subgroup of $G$, the order of $G_{1}^{\prime}$ is divisible by $p_{1} p_{2} \cdots p_{n}$ and contains no other prime factors besides $p_{1}, p_{2}, \cdots, p_{n}$. If now the order of $G_{1}$ is $g / p_{1} p_{2} \cdots p_{n}, G^{\prime}$ has an operator of order $p_{1} p_{2} \cdots p_{n}$ that is not in $G_{1}^{\prime}$ and that with $G_{1}^{\prime}$ generates $G^{\prime}$. If $A_{3}$ is an operator of $G$ that corresponds to this operator of $G^{\prime}, A_{3}$ is not commutative with any operator of $G_{1}$ that is not in $H$. The commutators formed by $A_{3}$ and two operators $A_{1}$ and $A_{2}$ of $G_{1}$ that correspond to different operators of $G_{1}^{\prime}$ are distinct. The number 'of commutators of $G$ is therefore equal to the order of $G_{1}^{\prime}$. If the order of $G$ is $g=g_{1} p_{1}^{a_{1}} p_{2}^{a_{2}} \cdots, p_{n}^{a_{n}}$, where $g_{1}$ is not divisible by $p_{1}, p_{2}, \cdots, p_{n}$, and if $G^{\prime}$ is of order $p_{1}^{a_{1}^{\prime}} p_{2}^{a_{2}^{\prime}} \cdots p_{n}^{a_{n}^{\prime}}$, then the number of commutators of $G$ is

$$
p_{1}^{a_{1}^{\prime}-1} p_{2}^{a_{2}^{\prime}-1} \cdots p_{n_{n}^{a^{\prime}-1}}^{a^{\prime}}
$$

The order of $H$ is $g_{1} p_{1}^{a_{1}-a_{1}^{\prime}} p_{2}^{a_{2}-a_{2}^{\prime}} \cdots p_{n}^{a_{n}-a_{n}^{\prime}}$. Therefore We have therefore $\alpha_{i}-\alpha_{i}^{\prime} \equiv \alpha_{i}^{\prime}-1 ; \quad \cdot \alpha_{i}^{\prime} \equiv \frac{1}{2}\left(\alpha_{i}+1\right) \quad(i=1,2, \cdots, n)$.

TheOREM IV.-If $G$ is a metabelian group of order $g=g_{1} p_{1}^{a_{1}} p_{2}^{a_{2}} \cdots p_{n}^{a_{n}}$, where $p_{1}, p_{2}, \cdots, p_{n}$ are distinct primes and $g_{1}$ is not divisible by $p_{1}, p_{2}, \cdots, p_{n}$, and if $G$ has a maximum abelian subgroup of order $g / p_{1} p_{2} \cdots p_{n}$, then ' $G^{\prime}$ is of order $p_{1}^{a_{1}^{\prime}} p_{2}^{a_{2}^{\prime}} \cdots p_{n}^{a_{n}^{\prime}}$, where

$$
1<\alpha_{i}^{\prime} \equiv \frac{1}{2}\left(\alpha_{i}+1\right) \quad(i=1,2, \cdots, n) .
$$

Suppose that $G$ has a maximum abelian subgroup of order

$$
g_{1} p_{1}^{a_{1}-a_{1}^{\prime}} p_{2}^{a_{2}-a_{2}^{\prime}} \cdots p_{n}^{a_{n}-a_{n}^{\prime}}
$$

and that $G^{\prime}$ is of order $p_{1}^{a_{1}-1} p_{2}^{a_{2}-1} \cdots p_{n}^{a_{n}-1}$. Consider the subgroup $P^{\prime}$ of $G^{\prime}$ of order $p_{i}^{a_{i}-1}(i=1,2, \cdots, n)$. Let $P_{1}^{\prime}$ be the subgroup of $P^{\prime}$ that is contained in $Q_{1}$. Now if any operator of $G$ that corresponds to an operator of $P_{1}^{\prime}$ is commutative with every operator of $G$ that corresponds to an operator of $P^{\prime}$ it is contained in $H$. Also, an operator of $G$ that corresponds to an operator of $P^{\prime}$ not in $P_{1}^{\prime}$ cannot be commutative with every operator of $G$ that corresponds to an operator of $P_{1}^{\prime}$. Now $P_{1}^{\prime}$, considered as a substitution group on the operators of the subgroup of $G$ that corresponds to $P^{\prime}$, is of degree 
and has

$$
g_{1} p_{1} p_{2} \cdots p_{i}^{a_{i}} \cdots p_{n}-g_{1} p_{1} p_{2} \cdots p_{i}^{a_{i}-a_{i}^{\prime}} \cdots p_{n}
$$

$$
g_{1} p_{1} p_{2} \cdots p_{i}^{a_{i}-1} \cdots p_{n}-g_{1} p_{1} p_{2} \cdots p_{i}^{a_{i}-a_{i}^{\prime}-1} \cdots p_{n}
$$

systems of intransitivity. The total number of letters in $P_{1}^{\prime}$ (considered as a substitution group) is therefore *

$$
\begin{aligned}
p_{i}^{a_{i}-a_{i}^{\prime}-1}\left(g_{1} p_{1} p_{2} \cdots p_{i}^{a_{i}} \cdots p_{n}\right. & -g_{1} p_{1} p_{2} \cdots p_{i}^{a_{i}-\alpha_{i}^{\prime}} \cdots p_{n} \\
& \left.\quad-g_{1} p_{1} p_{2} \cdots p_{i}^{a_{i}-1} \cdots p_{n}+g_{1} p_{1} p_{2} \cdots p_{i}^{a_{i}-a_{i}^{\prime}-1} \cdots p_{n}\right) .
\end{aligned}
$$

Now any operator of $G$ that corresponds to an operator of $P_{1}^{\prime}$ has $p_{i}$ conjugates and is commutative with $g_{1} p_{1} p_{2} \cdots p_{i}^{a_{i}-1} \cdots p_{n}$ operators of that subgroup of $G$ that corresponds to $P^{\prime}$. Therefore the total number of letters in $P_{1}^{\prime}$ is

$$
\left(p_{i}^{\alpha_{i}-a_{i}^{\prime}-1}-1\right)\left(g_{1} p_{1} p_{2} \cdots p_{i}^{\alpha_{2}} \cdots p_{n}-g_{1} p_{1} p_{2} \cdots p_{i}^{\alpha_{i}-1} \cdots p_{n}\right),
$$

and we have

$$
\begin{aligned}
& p_{1}^{a_{i} a_{i}^{\prime}-1}\left(g_{1} p_{1} p_{2} \cdots p_{i}^{a_{i}} \cdots p_{n}-g_{1} p_{1} p_{2} \cdots p_{i^{a_{i}} a_{i}^{\prime}} \cdots p_{n}-g_{1} p_{1} p_{2} \cdots p_{i^{i^{-1}}}^{a^{-1}} \cdots p_{n}\right. \\
& \left.+g_{1} p_{1} p_{2} \cdots p_{i}^{a_{i}-a_{i}^{\prime}-1} \cdots p_{n}\right) \\
& =\left(p_{i}^{a_{i}-a_{i}^{\prime}-1}-1\right)\left(g_{1} p_{1} p_{2} \cdots p_{i}^{a_{i}} \cdots p_{n}-g_{1} p_{1} p_{2} \cdots p_{i}^{a_{i}-1} \cdots p_{n}\right) \text {. } \\
& \alpha_{i}^{\prime}=\frac{1}{2}\left(\alpha_{i}-1\right) \text {. }
\end{aligned}
$$

Hence:

TheOREM V. If $G$ is a metabelian group of order $g=g_{1} p_{1}^{a_{1}} p_{2}^{a_{2}} \cdots p_{n}^{a_{n}}$, where $p_{1}, p_{2}, \cdots, p_{n}$ are distinct primes and $g_{1}$ is not divisible by $p_{1}, p_{2}, \cdots, p_{n}$, and if $G$ has a maximum abelian subgroup of order $g_{1} p_{1}^{a_{1}-a_{1}^{\prime}} p_{2}^{a_{2}-a_{2}^{\prime}} \cdots p_{n}^{a_{n}-a_{n}^{\prime}}$, its group of cogredient isomorphisms cannot be of order $p_{1}^{a_{1}-1} p_{2}^{a_{2}-1} \cdots p_{n}^{a_{n}-1}$ unless $\alpha_{i}^{\prime}=\frac{1}{2}\left(\alpha_{i}-1\right) \quad(i=1,2, \cdots, n)$.

If $G$ is any group of order $p^{m}$, where $p$ is a prime, it is known that it has an abelian subgroup of order $p^{\gamma}$, if

$$
\frac{\gamma(\gamma-1)}{2}<m . \dagger
$$

If now $G$ is a metabelian group, the minimum value of $m$ in order that $G$ necessarily contain an abelian subgroup of order $p^{\gamma}$ is less than this value when $\gamma>3$, as is shown in the following

TheOREM VI.-If $G$ is a metabelian group of order $p^{m}$, where $p$ is a prime it contains an abelian subgroup of order $p^{\gamma}$ if

$$
m>\frac{(\gamma+3)(\gamma-1)}{4} .
$$

*Frobenius, Crell e's Journal, vol. 101 (1887), p. 287.

† Millkr, Messenger of Mathematice, vol. 27 (1897-8), p. 120. 
Suppose that $G$ contains $p^{a}$ invariant operators and that its commutator subgroup is of order $p^{\beta}$. Let $A_{1}$ be a non-invariant operator of $G$. It has no more than $p^{\beta}$ conjugates and is therefore invariant in a $G_{1}$ of order $p^{m-\beta}$ that has at least $p^{a+1}$ invariant operators. Any non-invariant operator $A_{2}$ of $G_{1}$ is invariant in a $G_{2}$ of order $p^{m-2 \beta}$ that has at least $p^{a+2}$ invariant operators. By continuing this process, we see that $G_{\gamma-a-1}$ of order $p^{m-(\gamma-a-1) \beta}$ contains $p^{\gamma-1}$ invariant operators, if $m-(\gamma-\alpha-1) \beta>\gamma-1$. In this case $G_{\gamma-\alpha-1}$ contains an abelian subgroup of order $p^{\gamma}$. But $m-(\gamma-\alpha-1) \beta>\gamma-1$, if $m>\frac{1}{4}(\gamma+3)(\gamma-1)$, since $\beta \equiv \alpha$.

$\S 2$. The order of the product of two operators of a metabelian group.

Let $A$ and $B$ be any two operators of a metabelian group $G$, so that

$$
A^{-1} B A=h B, \quad A^{-1} B^{x} A=h^{x} B^{x},
$$

where $x$ is any integer. Then

$$
(A B)^{x}=h^{12 x(x-1)} A^{x} B^{x} .
$$

Suppose that $A$ and $B$ are of orders $\alpha$ and $\beta$ respectively, and let $\mu$ be the least common multiple of $\alpha$ and $\beta$. Then

$$
(A B)^{\mu}=h^{1 / 2 \mu(\mu-1)} A^{\mu} B^{\mu}=h^{1 / 2 \mu(\mu-1)} .
$$

Now the order of $h$ is a divisor of both $\alpha$ and $\beta$. Therefore if $\alpha$ and $\beta$ are both odd, or if they contain the factor 2 to different powers,

$$
(A B)^{\mu}=1 \text {. }
$$

But if $\alpha$ and $\beta$ contain the factor 2 to the same power, we cannot conclude that

In this case, however,

$$
(A B)^{\mu}=1 \text {. }
$$

$$
(A B)^{2 \mu}=1 \text {. }
$$

The operators $A \equiv b d, B \equiv a b \cdot c d \cdot e f g$ of the group $(a b c d)_{8}(e f g) c y c *$ may be cited as two operators of a metabelian group the order of whose product is twice the least common multiple of their orders.

Let $\gamma$ be the order of $C=A B$, and let $p^{a}, p^{b}, p^{c}$ be the highest powers of $p$ (any prime) contained in $\alpha, \beta, \gamma$ respectively. Then since $B C^{-1}=A^{-1}$ and $C^{-1} A=B^{-1}$, it follows from the preceding that of the numbers $a, b, c$ two are equal and the third one less than, or equal to, the other two, except that in the case $p=2$ the third one may be one greater than the value of the other two.

Hence :

* For this notation see CAYLeY, Quarterly Journal of Mathematics, vol. $25(1890-1)$, p. 76 . 
Theorem I.-If $G$ is a metabelian group, the order $\gamma$ of the product of any two of its operators $A$ and $B$ of orders $\alpha$ and $\beta$ respectively must be $a$ divisor of the least common multiple of $\alpha$ and $\beta$, except that when $\alpha$ and $\beta$ contain the same power of 2 as a factor, $\gamma$ is a divisor of twice the least common multiple of $\alpha$ and $\beta$. Also $\gamma$ must be a multiple of the least common multiple of $\alpha^{\prime}$ and $\beta^{\prime}$, where $\alpha=\delta \alpha^{\prime}, \beta=\delta \beta^{\prime}, \delta$ being the product of the powers of all the prime factors that occur to the same power in $\alpha$ and $\beta$, except that, when one of the numbers $\alpha, \beta$ contains the factor 2 to a power one higher than the other one does, $\gamma$ is a multiple of one-half the least common multiple of $\alpha^{\prime}$ and $\beta^{\prime}$.

Corollary. - If $\alpha$ and $\beta$ are relatively prime, $\alpha \beta$ is the order of $A B$.

This follows from the fact that in this case $A$ and $B$ are commutative.

CoRollary.-If $G$ is a metabelian group of order $p^{m}$, where $p$ is an odd prime, the order of the product of any two of its operators $A$ and $B$, of orders $p^{n_{1}}$ and $p^{n_{2}}$ respectively $\left(n_{1}>n_{2}\right)$, is $p^{n_{1}}$. If $p=2$, the order of $A B$ must be $2^{n_{1}}$, except that it may be $2^{n_{2}}$ when $n_{1}=n_{2}+1$.

If $G$ is an abelian group, the order of $A B$ is a multiple of the least common multiple of $\alpha^{\prime}$ and $\beta^{\prime}$, and a divisor of the least common multiple of $\alpha$ and $\beta$. Two commutative operators $A$ and $B$ can be chosen so that the order of $A B$ is any multiple of the least common multiple of $\alpha^{\prime}$ and $\beta^{\prime}$ that is also a divisor of the least common multiple of $\alpha$ and $\beta$. For, take

$$
A \equiv a_{1} a_{2} \ldots a_{\delta} \cdot b_{1} b_{2} \ldots b_{\delta^{\prime}} \cdot c_{1} c_{2} \cdots c_{a^{\prime}},
$$

where $\delta^{\prime}$ is any divisor of $\delta$, and

$$
B \equiv a_{\delta} a_{\delta-1} \cdots a_{2} a_{1} \cdot d_{1} d_{2} \cdots d_{\beta^{\prime}} .
$$

The order of $A B$ is $\delta^{\prime}$ times the least common multiple of $\alpha^{\prime}$ and $\beta^{\prime}$.

Theorem II.-In a metabelian group $G$ the order of the product of two operators that correspond respectively to two independent operators of $G^{\prime}$ is a multiple of the least common multiple of the orders of these operators of $G^{\prime}$.

This follows from the fact that the order of the product of two independent commutative operators is the least common multiple of the orders of the operators.

TheOREM III.-In a metabelian group $G$ of order $p^{m}$, where $p$ is a prime, the order of the product of two operators that correspond respectively to two operators of $G^{\prime}$ of different orders is equal to, or greater than, the greater of these orders.

This follows from the fact that the order of the product of two commutative operators of orders $p_{n_{1}}^{n_{1}}$ and $p^{n_{2}}$ respectively, where $n_{1}>n_{2}$, is $p^{n_{1}}$.

TheORem IV.-If $G$ is a metabelian group of order $p^{m}$, where $p$ is an odd 
prime, the $p^{\beta}$ power of all the operators form a group, $p^{\beta}$ being the highest order of any commutator of $G$.

For if $A^{p^{\beta}}=h_{1}$, and $B^{p^{\beta}}=h_{2}$, then

$$
(A B)^{p^{\beta}}=h^{1 / p^{\beta}\left(p^{\beta}-1\right)} A^{p^{\beta}} B^{p^{\beta}}=h_{1} h_{2},
$$

where $A^{-1} B A=h B$.

\section{§3. The commutators of a metabelian group.}

The commutator subgroup of a group is invariant in the group,* and in a group of order $p^{m}$ an operator of order $p$ cannot be transformed into one of its own powers, except the first. $\dagger$ Hence, a group of order $p^{m}$, where $p$ is a prime, is metabelian if its commutator subgroup is of order $p$.

If the commutator subgroup of a group $G$ of order $p^{m}$ is of order $p^{a}$ and contains a subgroup $H_{1}$ of order $p^{a-1}$ that is invariant in $G$, then $G / H_{1}$ is met. abelian, since its commutator subgroup is of order $p$.

Let $G$ be a metabelian group whose group of cogredient isomorphisms is generated by three independent operators. Let $A_{1}, A_{2}, A_{3}$ be three operators of $G$ that correspond respectively to the three generators $A_{1}^{\prime}, A_{2}^{\prime}, A_{3}^{\prime}$, of $G^{\prime}$. If

$$
A_{2}^{-1} A_{1} A_{2}=h_{1} A_{1}, \quad A_{3}^{-1} A_{1} A_{3}=h_{2} A_{1}, \quad A_{3}^{-1} A_{2} A_{3}=h_{3} A_{2},
$$

then $h_{1}, h_{2}, h_{3}$ generate the commutator subgroup of $G$. We proceed to prove that every operator of this subgroup is a commutator.

This is done by showing that integral values of $a_{1}, a_{2}, a_{3}, b_{1}, b_{2}, b_{3}$ can be found that will satisfy the relation

$$
\left(A_{1}^{a_{1}} A_{2}^{a_{2}} A_{3}^{a_{3}}\right)^{-1} \cdot A_{1}^{b_{1}} A_{2}^{b_{2}} A_{3}^{b_{3}} \cdot\left(A_{1}^{a_{1}} A_{2}^{a_{2}} A_{3}^{a_{3}}\right)=h_{1}^{a_{1}} h_{2}^{a_{2}} h_{3}^{a_{3}} A_{1}^{b_{1}} A_{2}^{b_{2}} A_{3}^{b_{3}}
$$

for all integral values of $\alpha_{1}, \alpha_{2}, \alpha_{3}$ taken modulo the orders of $h_{1}, h_{2}, h_{3}$ respectively.

Now

$$
\begin{aligned}
& \left(A_{1}^{a_{1}} A_{2}^{a_{2}} A_{3}^{a_{3}}\right)^{-1} \cdot A_{1}^{b_{1}} A_{2}^{b_{2}} A_{3}^{b_{3}} \cdot\left(A_{1}^{a_{1}} A_{2}^{\grave{a}_{2}} A_{3}^{a_{3}}\right) \\
& \quad=h_{3}^{a_{3} b_{2}} h_{2}^{a_{3} b_{1}} h_{3}^{-a_{2} b_{3}} h_{1}^{a_{2} b_{1}} h_{2}^{-a_{1} b_{3}} h_{1}^{-a_{1} b_{2}} A_{1}^{b_{1}} A_{2}^{b_{2}} A_{3}^{b_{3}} \cdot
\end{aligned}
$$

Hence the relation given above will be satisfied if the following equations are satisfied

$$
a_{2} b_{1}-a_{1} b_{2}=\alpha_{1}, \quad a_{3} b_{1}-a_{1} b_{3}=\alpha_{2}, \quad a_{3} b_{2}-a_{2} b_{3}=\alpha_{3} .
$$

But integral values of $a_{1}, a_{2}, a_{3}, b_{1}, b_{2}, b_{3}$ can always be found that satisfy these equations.

* Miller, Quarterly Journal of Mathematics, vol. 28 (1896), p. 266 . Cf. FrobeNius, Berliner Sitzungeberichte, 1896, p. 1348.

† Frobenius, loc. cit., 1895, p. 982. 
If $h_{1}, h_{2}, h_{3}$ are not all distinct, or if any of them equal identity, the conclusion still holds.

Consider now a metabelian group $G$ of order $p^{m}$ whose group of cogredient isomorphisms is generated by four independent operators, $A_{1}^{\prime}, A_{2}^{\prime}, A_{3}^{\prime}, A_{4}^{\prime}$, and let $A_{1}, A_{2}, A_{3}, A_{4}$ be operators of $G$ that correspond respectively to these generators of $G^{\prime}$. Let

$$
\begin{array}{lll}
A_{2}^{-1} A_{1} A_{2}=h_{1} A_{1}, & A_{3}^{-1} A_{1} A_{3}=h_{2} A_{1}, & A_{4}^{-1} A_{1} A_{4}=h_{3} A_{1}, \\
A_{3}^{-1} A_{2} A_{3}=h_{4} A_{2}, & A_{4}^{-1} A_{2} A_{4}=h_{5} A_{2}, & A_{4}^{-1} A_{3} A_{4}=h_{6} A_{3} .
\end{array}
$$

We assume that it is possible that the $h_{i}(i=1,2, \ldots, 6)$ be all different from identity and independent. We shall justify this assumption later.

Now the $h_{i}(i=1,2, \cdots, 6)$ generate the commutator subgroup of $G$; that is, every commutator is obtained from $A_{1}^{a_{1}} A_{2}^{a_{2}} A_{3}^{a_{3}} A_{4}^{a_{4}}$ and $A_{1}^{b_{1}} A_{2}^{b_{2}} A_{3}^{b_{3}} A_{4}^{b_{4}}$, where $a_{1}, a_{2}, a_{3}, a_{4}$, and also $b_{1}, b_{2}, b_{3}, b_{4}$ take all possible values modulo the orders of $A_{1}^{\prime}, A_{2}^{\prime}, A_{3}^{\prime}, A_{4}^{\prime}$ respectively.

We have

$$
\begin{aligned}
& \left(A_{1}^{a_{1}} A_{2}^{a_{2}} A_{3}^{a_{3}} A_{4}^{a_{4}}\right)^{-1} A_{1}^{b_{1}} A_{2}^{b_{2}} A_{3}^{b_{3}} A_{4}^{b_{4}}\left(A_{1}^{a_{1}} A_{2}^{a_{2}} A_{3}^{a_{3}} A_{4}^{\alpha_{4}}\right) \\
& \quad=h_{1}^{a_{2} b_{1}-a_{1} b_{2}} h_{2}^{a_{3} b_{1}-a_{1} b_{3}} h_{3}^{a_{a} b_{1}-a_{1} b_{4}} h_{4}^{a_{3_{2} b_{2}-a_{2} b_{3}}} h_{5}^{a_{4} b_{2}-a_{2} b_{4}} h_{6}^{a_{4} b_{3}-a_{3} b_{4}} A_{1}^{b_{1}} A_{2}^{b_{2}} A_{3}^{b_{3}} A_{4}^{b_{4}} .
\end{aligned}
$$

If now $p^{m_{i}}$ is the order of $h_{i}$, and $k_{i}$ is an integer $(i=1,2, \ldots, 6)$, then $h_{1}^{a_{1}} h_{2}^{a_{2}} h_{3}^{a_{3}} h_{4}^{a_{4}} h_{5}^{a_{5}} h_{6}^{a_{6}}$ is an operator of the commutator subgroup of $G$ that is not a commutator if integral values of $a_{i}$ and $b_{j}(i, j=1,2, \ldots, 4)$ cannot be found to satisfy the following equations :

$a_{2} b_{1}-a_{1} b_{2}=\alpha_{1}+k_{1} p^{m_{1}}, a_{3} b_{1}-a_{1} b_{3}=\alpha_{2}+k_{2} p^{m_{2}}, a_{4} b_{1}-a_{1} b_{4}=\alpha_{3}+k_{3} p^{m_{3}}$, $a_{3} b_{2}-a_{2} b_{3}=\alpha_{4}+k_{4} p^{m_{4}}, a_{4} b_{2}-a_{2} b_{4}=\alpha_{5}+k_{5} p^{m_{5}}, a_{4} b_{3}-a_{3} b_{4}=\alpha_{6}+k_{6} p^{m_{6}}$.

Such integral values cannot be found to satisfy these equations for all values of $\alpha_{i}(i=1,2, \ldots, 6)$; e. g., for $\alpha_{i}=1$. Therefore if a group exists that has the properties assumed, the commutator subgroup of this group contains operators that are not commutators. The group generated by the following operators is such a group : *

$$
\begin{gathered}
h_{1} \equiv a c \cdot b d, \quad h_{2} \equiv e g \cdot f h, \quad h_{3} \equiv i k \cdot j l \\
h_{4} \equiv m o \cdot n p, \quad h_{5} \equiv q s \cdot r t, \quad h_{6} \equiv u w \cdot v x \\
A_{1} \equiv a c \cdot e g \cdot i k, \quad A_{2} \equiv a b \cdot c d \cdot m o \cdot q s \\
A_{3} \equiv e f \cdot g h \cdot m n \cdot o p \cdot u w, \quad A_{4} \equiv i j \cdot k l \cdot q r \cdot s t \cdot u v \cdot w x .
\end{gathered}
$$

* This group was constructed by Professor Miller. 
The order of this group is 1024 . The commutator subgroup is of order 64 . It contains 36 commutators and 28 operators that are not commutators.

If $h_{5}=h_{6}=1$, that is, if $A_{4}$ is commutative with $A_{2}$ and $A_{3}$, the commutator subgroup is unchanged except that $h_{5}$ and $h_{6}$ are replaced by identity. Now $h_{3} h_{4}$ is not a commutator in the original group. Moreover no commutator of the original group reduces to $h_{3} h_{4}$ when $h_{5}$ and $h_{6}$ are replaced by identity. Hence if there is a group with these properties, its conmutator subgroup contains operators that are not commutators. Such a group is generated by the following operators :

$$
\begin{gathered}
h_{1} \equiv a c \cdot b d, \quad h_{2} \equiv e g \cdot f h, \quad h_{3} \equiv i k \cdot j l, \quad h_{4} \equiv m o \cdot n p \\
A_{1} \equiv a c \cdot e g \cdot i k, \quad A_{2} \equiv a b \cdot c d \cdot m o, \quad A_{3} \equiv e f \cdot g h \cdot m n \cdot o p, \quad A_{4} \equiv i j \cdot k l .
\end{gathered}
$$

The order of this group is 256. Its commutator subgroup is of order 16 and contains only 15 commutators.

\section{§4. Metabelian groups whose commutator subgroups are cyclic.}

Let $G$ be a metabelian group of order $p^{m}$, where $p$ is a prime. Suppose that the commutator subgroup of $G$ is cyclic of order $p^{a}$ and that $A_{1}, A_{2}, \ldots, A_{n}$ are operators of $G$ corresponding respectively to the independent generators of $G^{\prime}$ that are of the highest order $p^{a_{1}}$. No one of these, as $A_{1}$, can be commutative with all the others, for in that case $A_{1}$ would be invariant in the subgroup of $G$ corresponding to the subgroup of $G^{\prime}$ that is generated by the generators of highest order in $G^{\prime}$. Hence no commutator of highest order in $G$ could be formed by $A_{1}$ and any operator of $G$. But such a commutator can always be formed by $A_{1}$ and some operator of $G$.

We may assume therefore that $A_{1}^{-1} A_{2} A_{1}=h A_{2}$, where $h$ is a commutator of $G$ of order $p^{a_{1}}$. We can choose for $A_{3}, A_{4}, \cdots, A_{n}$ operators that are commutative with $A_{1}$ and $A_{2}$. For if $A_{1}^{-1} A_{3} A_{1}=h_{1} A_{3}, h_{1}$ must be a power of $h$, say $h_{1}=h^{a}$, since the commutator subgroup is cyclic. Now

$$
A_{1}^{-1} \cdot A_{2}^{p^{a_{1}-a}} A_{3} \cdot A_{1}=A_{2}^{p^{a_{1}-a}} A_{3} \equiv B_{3},
$$

and we can take the operator $B_{3}^{\prime}$ of $G^{\prime}$ that corresponds to $B_{3}$ as a generator in place of $A_{3}^{\prime}$. If $A_{2}^{-1} B_{3} A_{2}=h_{2} B_{3}$, then $h_{2}=h^{b}$ and

$$
A_{2}^{-1} \cdot A_{1}^{b-p^{a_{1}}} B_{3} \cdot A_{2}=A_{1}^{b-p^{a_{1}}} B_{3} \equiv C_{3} .
$$

In place of $B_{3}^{\prime}$ we can take $C_{3}^{\prime}$ as one of the independent generators of $G^{\prime}$ of order $p^{\alpha_{1}}$. Both $A_{1}$ and $A_{2}$ are commutative with $C_{3}$. Similarly, if $A_{4}, \cdots, A_{n}$ are not commutative with $A_{1}$ and $A_{2}$, we can choose in place of them operators that are. Of these we can find two whose commutator is of 
order $p^{a_{1}}$, and then choose all the others so that they are commutative with these two and with $A_{1}$ and $A_{2}$. Proceeding in this way, we can arrange the generators of $G^{\prime}$ of order $p^{a_{1}}$ in pairs. If there should be an odd number of these generators, an operator of $G$ corresponding to one of them would be commutative with every operator of $G$ that corresponds to an independent generator of $G^{\prime}$ of order $p^{a_{1}}$. It has been shown that this is impossible.

By similar reasoning it can be shown that the independent generators of $G^{\prime}$ can be so chosen that any operator of $G$ corresponding to an independent generator of $G^{\prime}$ of any order is commutative with those operators of $G$ that correspond to the independent generators of $G^{\prime}$ of any higher order, and is not commutative with all the operators of $G$ that correspond to independent generators of $G^{\prime}$ of the same order. We have therefore

TheOREM I.-If the commutators of a metabelian group of order $p^{m}$, where $p$ is a prime, form a cyclic group, the number of independent generators of any given order of $G^{\prime}$ is even.*

Consider a metabelian group $G \equiv\left\{Q, A_{1}, A_{2}\right\}$ whose invariant operators form the cyclic group $\{Q\}$ of order $p^{\alpha}$ and whose group of cogredient isomorphisms is of order $p^{2 \beta}$ of the type $(\beta, \beta)$. We can assume that

If $i=0$, the relations

$$
A_{1}^{p^{\beta}}=A_{2}^{p^{\beta}}=Q^{p^{\beta-i}} \quad(i=0,1,2, \cdots, \beta) .
$$

$$
A_{1}^{p^{\beta}}=A_{2}^{p^{\beta}}=1
$$

define the same group as the relations just given.

There are accordingly no more than $\beta+1$ types of metabelian groups whose invariant operators form a cyclic group of order $p^{a}$ and whose groups of cogredient isomorphisms are of order $p^{2 \beta}$ of the type $(\beta, \beta)$. These $\beta+1$ types may be defined as follows:

$$
\begin{aligned}
& Q^{p^{\alpha}}=1, A_{1}^{p^{\beta}}=A_{2}^{p^{\beta}}=Q^{p^{\beta-i}} \quad(i=0,1,2, \cdots, \beta), \\
& A_{1}^{-1} A_{2} A_{1}=A_{2} Q^{p^{a-\beta}}, \quad A_{1} Q=Q A_{1}, \quad A_{2} Q=Q A_{2} .
\end{aligned}
$$

These relations in every case define a group, since substitution groups can be constructed that satisfy them. Moreover these groups are distinct.

If $G$ is a metabelian group of order $p^{m}$, where $p$ is a prime, whose commutator subgroup is cyclic and whose group of cogredient isomorphisms is of the type $(\beta, \beta, \cdots$, with $2 s$ terms), $s$ being any positive integer, there always exists a metabelian group of order $p^{m+2 \beta}$ whose invariant operators are the same as those of $G$ and whose group of cogredient isomorphisms is of the type $(\beta, \beta, \cdots$, with $2 s+2$ terms). For, if $G$ be represented as a regular substitu-

* Cf. Young, A merican Journal of Mathematics, vol. 15 (1893), p. 171 ; Burnside, Theory of Groups of Finite Order, 1897, p. 68. 
tion group and $G_{1}$ is the simply isomorphic group all of whose substitutions are commutative with every substitution of $G^{*}$, and if $A_{1}$ and $A_{2}$ are a pair of generators of $G$ such as are described in the preceding discussion, and $B_{1}$ and $B_{2}$ the corresponding substitutions of $G_{1},-$ then $G_{2} \equiv\left\{G, B_{1}, B_{2}\right\}$ is a metabelian group with the same invariant operators and the same commutators that $G$ has, and the group of cogredient isomorphisms of $G_{2}$ is of the type ( $\beta, \beta, \cdots$, with $2 s+2$ terms). We have just shown that $\beta+1$ metabelian groups exist whose invariant operators form a cyclic group of order $p^{\alpha}, \alpha$ being any positive integer, and whose groups of cogredient isomorphisms are of the type $(\beta, \beta)$, where $\beta \equiv \alpha$. Therefore metabelian groups exist whose invariant operators form a cyclic group of order $p^{\alpha}$ and whose groups of cogredient isomorphisms are of the type $(\beta, \beta, \ldots$ with $2 s$ terms), $s$ being any positive integer.

Let $G$ be such a group, and let $A_{1}, A_{2} ; A_{3}, A_{4}$ be two pairs of generators such as have been described. If

$$
\begin{array}{cl}
A_{1}^{p^{\beta}}=A_{2}^{p^{\beta}}=Q^{p^{\beta-i}}, & A_{3}^{p^{\beta}}=A_{4}^{p^{\beta}}=Q^{p^{\beta-j}} \\
A_{2}^{-1} A_{1} A_{2}=A_{1} Q^{p^{\alpha-\beta}}, & A_{4}^{-1} A_{3} A_{4}=A_{3} Q^{p^{\alpha-\beta}}, \\
A_{1} A_{3}=A_{3} A_{1}, \quad A_{1} A_{4}=A_{4} A_{1}, & A_{2} A_{3}=A_{3} A_{2}, \quad A_{2} A_{4}=A_{4} A_{2},
\end{array}
$$

then in place of $A_{1}, A_{2}, A_{3}, A_{4}$ we can take the following:

$$
B_{1} \equiv A_{3}^{1-p j-i} A_{1}, \quad B_{2} \equiv A_{3}^{1-p j-i} A_{2}, \quad B_{3} \equiv A_{3},
$$

and, according as $p$ is odd or even,

or

$$
B_{4} \equiv A_{1}^{-p^{\beta-p^{j-i}+1}} A_{2}^{p^{\beta}+p^{j-i}-1} A_{4},
$$

where

$$
B_{4} \equiv A_{1}^{-p^{\beta}-p^{j-1}+1} A_{2}^{p^{\beta}+p^{j-i}-1} A_{3}^{x} A_{4},
$$

$$
x\left(1+p^{a-\beta+j-1}\right) \equiv p^{a-\beta+j-i}\left(\bmod p^{a-\beta+j}\right) .
$$

Either of these pairs generates with $\{Q\}$ a group that is simply isomorphic with $\left\{Q, A_{3}, A_{4}\right\}$. Also $B_{1}$ and $B_{2}$ are commutative with $B_{3}$ and $B_{4}$, and

$$
B_{2}^{-1} B_{1} B_{2}=B_{1} Q^{p a-\beta}, \quad . B_{4}^{-1} B_{3} B_{4}=B_{3} Q^{p-\beta} \text {. }
$$

Therefore the subgroups generated by $\{Q\}$ and the respective pairs of generators may be assumed to be all of one type and we have

TheOREM II.-There are exactly $\beta+1$ groups of order $p^{a+2 \beta s}$, where $p$ is a prime, $\alpha, \beta$, and $s$ positive integers, and $\beta \equiv \alpha$, whose invariant operators

* Jordan, Journal de l'Ecole Polytechnique, vol. 22 (1861), p. 153. 
form a cyclic group of order $p^{a}$ and whose groups of cogredient isomorphisms are abelian of the type

$$
(\beta, \beta, \cdots \text { with } 2 s \text { terms }) .^{*}
$$

Suppose that the invariant operators of a group $G$ form a cyclic group $\{Q\}$ of order $p^{\alpha}$, where $p$ is a prime, and that $G^{\prime \prime}$ is abelian of the type $\left(\alpha_{1}, \alpha_{1}, \cdots\right.$ with $2 s_{1}$ terms; $\alpha_{2}, \alpha_{2}, \cdots$ with $2 s_{2}$ terms; $\cdots$;

$$
\alpha_{n}, \alpha_{n}, \ldots, \text { with } 2 s_{n} \text { terms), }
$$

where $\alpha_{1}<\alpha_{2}<\cdots<\alpha_{n-1}<\alpha_{n} \equiv \alpha$. We know that there are $\alpha_{i}+1$ metabelian groups whose invariant operators form a cyclic group of order $p^{\alpha}$ and whose groups of cogredient isomorphisms are of the type

$$
\left(\alpha_{i}, \alpha_{i}, \cdots \text { with } 2 s_{i} \text { terms }\right) \quad(i=1,2, \cdots, n) .
$$

We shall represent these $\alpha_{i}+1$ groups by the symbols

$$
G_{0, i}, G_{1, i}, \cdots, G_{a_{i}, i} \text {. }
$$

We write down the following scheme in which the groups of the $i$-th column are the $\alpha_{i}+1$ groups whose groups of cogredient isomorphisms are of the type

$$
\begin{aligned}
& \left(\alpha_{i}, \alpha_{i}, \ldots \text { with } 2 s_{i} \text { terms }\right): \\
& G_{0,1}, G_{0,2}, \cdots, G_{0, i}, \cdots, G_{0, n} \\
& G_{1,1}, G_{1,2}, \ldots, G_{1, i}, \cdots, G_{1, n} \\
& G_{a_{1}, 1}, G_{a_{1}, 2}, \cdots, G_{a_{1}, i}, \cdots, G_{a_{1}, n} \\
& G_{a_{2}, 2}, \cdots, \quad G_{a_{2}, i}, \cdots, G_{a_{2}, n} \\
& G_{a_{i}, i}, \cdots, \quad G_{a_{i}, n} \\
& G_{a_{n}, n} .
\end{aligned}
$$

All the groups in this scheme have the same invariant operators, but no two of the groups have any non-invariant operators in common. The groups in the

* This result does not agree with that obtained by Bursside, loc. cit., p. 69. For the case $\beta=1$, see Young, loc. cit., pp. 168-177. 
$j$ th row $\left(j=1,2, \ldots, a_{n}^{+1}\right)$ contain operators of order $p^{a+j-1}$ but none of higher order.* Any group $G$ whose invariant operators form a cyclic group of order $p^{\alpha}$ and whose group of cogredient isomorphisms is abelian of the type given above can be generated by groups in this scheme, one group being taken from each column. Any group generated in this way is of the type under consideration. It is assumed that all the operators of any group in any column of this scheme are commutative with all the operators of all the groups contained in all the other columns.

We now consider how many distinct groups can be obtained in this way; that is, how many distinct groups are contained in the scheme

$$
\left\{G_{i_{1}, 1}, G_{i_{2}, 2}, \cdots, G_{i_{n}, n}\right\},
$$

where $i_{j}=0,1,2, \ldots, \alpha_{j}$, and $j=1,2, \ldots, n$.

In the first place, $G_{i, j}$ and $G_{k, l}$, where $k<i$ and $l>j$, generate the same group as $G_{i, j}$ and $G_{i, l}$. For if $A$ is an operator of order. $p^{a+i}$ in $G_{i, j}$ corresponding to an independent generator of $G_{i, j}^{\prime}$ of order $p^{a_{j}}$, and $B$ is an operator of order $p^{a+k}$ in $G_{k, l}$ corresponding to an independent generator of $G_{k, l}^{\prime}$ of order $p^{a_{l}}$, then $A B$ is an operator of $\left\{G_{i, j}, G_{k, l}\right\}$ of order $p^{a+i}$ and corresponds to an operator of order $p^{a_{l}}$ in the group of cogredient isomorphisms of $\left\{G_{i, j}, G_{k, l}\right\}$. This latter may be taken as one of the independent generators of order $p^{a_{l}}$ of this group of cogredient isomorphisms.

We can take for $B$ in turn operators of order $p^{a+k}$ corresponding respectively to the independent generators of order $p^{a_{l}}$ of $G_{k, l}^{\prime}$, and each time we get an operator of $\left\{G_{i, j}, G_{k, l}\right\}$ of order $p^{a+i}$ corresponding to an independent generator of the group of cogredient isomorphisms of $\left\{G_{i, j}, G_{k, l}\right\}$ of order $p^{a_{l}}$. Therefore $\left\{G_{i, j}, G_{k, l}\right\} \equiv\left\{G_{i, j}, G_{i, l}\right\}$.

Suppose now that $A$ is an operator of order $p^{a+i}$ in $G_{i, j}$ corresponding to an independent generator of $G_{i, j}^{\prime}$ of order $p^{a_{j}}$, and that $B$ is an operator of order $p^{a+i+x}$ in $G_{i+x, k}$ corresponding to an independent generator of $G_{i+x, k}^{\prime}$ of order $p^{a_{k}}$, where $k>j$. Then $A B^{p^{a_{k}-a_{j}}}$ corresponds to an operator of order $p^{a_{j}}$ in the group of cogredient isomorphisms of $\left\{G_{i, j}, G_{i+x, k}\right\}$. This may be taken as one of the independent generators of order $p^{a_{j}}$ in this group of cogredient isomorphisms. Now $B^{p^{a_{k}-a_{j}}}$ is of order $p^{a+i+x-a_{k}+a_{j}}$ and the order of $A B^{a^{a_{k}-a_{j}}}$ is $p^{a+i+x-\alpha_{k}+\alpha_{j}}$, if $x>\alpha_{k}-\alpha_{j}$. It follows, therefore, if $x>\alpha_{k}-\alpha_{j}$, that

$$
\left\{G_{i, j}, G_{i+x, k}\right\} \equiv\left\{G_{i+x-\left(a_{k}-a_{j}\right), j}, G_{i+x, k}\right\} .
$$

Hence in order to get all the distinct groups in the scheme

$$
\left\{G_{i_{1}, 1}, G_{i_{2}, 2}, \cdots, G_{i_{n}, n}\right\}
$$

* If $p=2, G_{0, n}$ contains operators of order $p^{\alpha+1}$. Some of the statements in the following argument would have to be modified to cover this case, but the final result, as stated in Theorem III, is the same whether $p$ is even or odd. 
we need to combine with $G_{i_{x}, x}$ only $\alpha_{x+1}-\alpha_{x}+1$ groups of the type $G_{i_{x+1}, x+1}$ namely, those for which

$$
0 \equiv i_{x+1}-i_{x} \equiv \alpha_{x+1}-\alpha_{x} .
$$

Therefore the number of distinct groups in the scheme under consideration is equal to, or less than,

$$
\left(\alpha_{1}+1\right)\left(\alpha_{2}-\alpha_{1}+1\right) \cdots\left(\alpha_{n}-\alpha_{n-1}+1\right) .
$$

Now, these groups are all distinct, since any two must differ in at least one of the components, say that one contains $G_{i, j}$ and that the other contains $G_{i^{\prime}, j}$, where $i^{\prime}>i$; and the one has no operator of order greater than $p^{a+i}$ corresponding to an independent generator of order $p^{a_{j}}$ in the group of cogredient isomorphisms, while the other has such an operator of order $p^{a+i^{\prime}}$.

The relations under consideration always define a group since substitution groups can be constructed that satisfy these relations. We can therefore enunciate the following

TheOREM III.-The number of metabelian groups whose invariant operators form a cyclic group of order $p^{a}$, where $p$ is a prime, and whose groups of cogredient isomorphisms are of order $p^{2\left(a_{1} s_{1}+a_{2} s_{2}+\ldots+a_{n} s_{n}\right)}$ of the type

$\left(\alpha_{1}, \alpha_{1}, \ldots\right.$ with $2 s_{1}$ terms, $\alpha_{2}, \alpha_{2}, \ldots$ with $2 s_{2}$ terms, $\ldots$,

$$
\alpha_{n}, \alpha_{n}, \ldots \text { with } 2 s_{n} \text { terms), }
$$

where $\alpha_{1}<\alpha_{2}<\cdots<\alpha_{n-1}<\alpha_{n} \equiv \alpha$, and $s_{1}, s_{2}, \cdots s_{n}$ are any positive integers, is

$$
\left(\alpha_{1}+1\right)\left(\alpha_{2}-\alpha_{1}+1\right) \cdots\left(\alpha_{n}-\alpha_{n-1}+1\right) \text {. }
$$

§5. Metabelian groups whose invariant operators form a group that is the direct product of two cyclic groups.

We shall now consider the number of metabelian groups of order $p^{m}, p$ being a prime, whose invariant operators form a subgroup that is the direct product of two cyclic groups of orders $p^{a_{1}}$ and $p^{a_{2}}$ respectively, and whose groups of cogredient isomorphisms are of order $p^{2 \beta}$ and of type $(\beta, \beta)$. It is assumed that

$$
\alpha_{1}-\beta \equiv \alpha_{2}, \quad \beta \equiv \alpha_{2},
$$

and that the commutator subgroup is contained in the cyclic subgroup of order $p^{a_{1}}$ formed by invariant operators.

The subgroup $H$ formed by the invariant operators has the independent generators $Q, R$, of orders $p^{a_{1}}$ and $p^{a_{2}}$ respectively. The entire group $G$ is gen- 
erated by $H, A, B$, where $A$ and $B$ are operators of $G$ corresponding to independent generators of $G^{\prime}$ of order $p^{\beta}$. We can always choose $A$ and $B$ so that $A^{p^{\beta}}$ is contained in $\{Q\}$ and $B^{p^{\beta}}$ is not contained in $\{Q\}$, unless $B^{p^{\beta}}=A^{p^{\beta}}$. Therefore all the groups under consideration can be represented by the symbol ${ }_{k} G_{i, j}\left(k=0,1,2, \cdots, \beta ; i=0,1, p, p^{2}, \cdots, p^{\beta-1} ; j=0,1, p, p^{2}, \cdots, p^{a_{2}-1}\right)$. The subscript $k$, at the left, indicates that $A^{p^{\beta}}=Q^{p^{\beta-k}}$, and the subscripts $i, j$, at the right indicate that $B^{p^{\beta}}=Q^{i} R^{j}$. If $i>p^{\beta-1}$, we can take

$$
B_{1} \equiv Q^{-i \mid p^{\beta}} B
$$

for $B$. Then $B_{1}^{{ }^{\beta}}=R^{j}$. If $k=0$, we have $A^{p^{\beta}}=Q^{p^{\beta}}$. This gives the group in which $A^{p^{\beta}}=1$, since if we take $A_{1} \equiv Q^{-1} A$ for $A$, then $A_{1}^{{ }^{\beta}}=1$. When $j=0$, we may take $i=p^{\beta-k}$.

These relations in every case define a group, since substitution groups can be constructed that satisfy them.

We have now to consider how many distinct groups are represented by ${ }_{k} G_{i},{ }_{i}$. The following identities can be established:

$$
\begin{array}{rlrl}
{ }_{k} G_{i, j} & \equiv{ }_{k} G_{0, j}, & \text { when } & p^{\beta-k} \equiv i \equiv p^{\beta-1}, j>0 ; \\
{ }_{k} G_{i, j} \equiv{ }_{0} G_{i, j}, & \text { when } & \frac{p^{\beta-k}}{i} \equiv \frac{p^{a_{2}}}{j}, i>0, j>0 ; \\
{ }_{0} G_{p^{l}, j} \equiv{ }_{\beta-l} G_{0,0}, & \text { when } & j \equiv p^{l} ; \\
{ }_{k} G_{i, j} \equiv{ }_{k^{\prime}} G_{0, j^{\prime}}, & \text { when } & i=p^{\beta-k^{\prime}}, j^{\prime}=\frac{j p^{\beta-k}}{i}, i \equiv j .
\end{array}
$$

By considering the orders of their operators it can be shown that those groups are distinct for which the following relations hold:

$$
\begin{gathered}
\frac{p^{\beta-k}}{i}<\frac{p^{\alpha_{2}}}{j}, \quad \text { when } \quad i>0, \quad j>0, \quad k>0 ; \\
i<p^{\beta-k}, \quad \text { when } \quad j>0 ; \\
j<i, \quad \text { when } \quad i>0, \quad j>0 ; \\
i=p^{\beta-k}, \quad k>0, \quad \text { or } \quad i=k=0, \quad \text { when } \quad j=0 .
\end{gathered}
$$

It follows from the above that there is no other distinct group ${ }_{k} G_{i, j}$. The number of groups ${ }_{k} G_{i, j}$ satisfying these relations is

$$
N=\beta+1+\frac{\alpha_{2}\left(5+3 \beta+3 \alpha_{2} \beta-2 \alpha_{2}^{2}\right)}{6} .
$$


We can state this result as follows :

Theorem I.-There are

$$
\beta+1+\frac{\alpha_{2}\left(5+3 \beta+3 \alpha_{2} \beta-2 \alpha_{2}^{2}\right)}{6}
$$

metabelian groups of order $p^{a_{1}+a_{2}+2 \beta}$, where $p$ is a prime, $\alpha_{1}-\beta \equiv \alpha_{2}, \beta \equiv \alpha_{2}$, whose groups of cogredient isomorphisms are of order $p^{2 \beta}$ and of the type $(\beta, \beta)$, and whose invariant operators form a group that is the direct product of an operator of order $p^{a_{1}}$ and one of order $p^{a_{2}}$, the commutators being powers of the operator of order $p^{a_{1}}$.

§6. Groups that have metabelian groups of cogredient isomorphisms.

If we form the group of cogredient isomorphisms $G^{\prime}$ of any group $G$, then the group of cogredient isomorphisms $G^{\prime \prime}$ of $G^{\prime}$, and so on, we finally come either to identity or to a group that has no invariant operators except identity, and that is therefore simply isomorphic with its group of cogredient isomorphisms. In this section the groups for which this process leads to identity are classified according to the number of these successive groups of cogredient isomorphisms. Thus, abelian groups are of the first class ; metabelian groups are of the second class; and groups that have metabelian groups of cogredient isomorphisms are of the third class.

Let $G$ be a group of the third class. If the order of $G$ is $p_{1}^{a_{1}} p_{2}^{a_{2}} \cdots p_{n}^{a_{n}}$, where $p_{1}, p_{2}, \ldots, p_{n}$ are distinct primes, $G$ is the direct product of groups of orders $p_{1}^{a_{1}}, p_{2}^{a_{2}}, \ldots, p_{n}^{a_{n}}$ respectively (see introduction). The class of each of these factor groups is not greater than 3. Every subgroup (and likewise every quotient group) of $G$ is of the first, second, or third class. If a group is the direct product of factor groups whose orders are powers of distinct primes, and if the order of the group is not divisible by the fourth power of a prime, every one of these factor groups is of the first or second class, and the group itself is of the first or second class. Hence the order of every group of the third class is divisible by the fourth power of a prime.

Since a metabelian group is characterized by the property that all of its commutators are invariant (see introduction), it follows that a group of the third class is characterized by the property that those of its commutators formed by any of its commutators and any operator of the group are invariant.

If $G$ is a group of the $n$th class and if $A$ is an operator of $G$ that corresponds to an invariant operator of $G^{\prime}$, then $A^{-1} C A=C h, C$ being any operator of $G$, and $h$ an invariant commutator of $G$. If $\alpha$ is the order of the operator of $G^{\prime}$ that corresponds to $A$, then $A^{-a} C A^{a}=C h^{a}=C$. If now $C$ runs through all the operators of $G, h$ will run through a set of invariant commutators of $G$ and $\alpha$ will be the order of some one of these. Hence the 
order of every invariant operator of $G^{\prime}$ is the order of some invariant commutator of $G$.

If $t$ is any non-invariant commutator of a group $G$ of the third class, and if $B^{-1} C B=C t, B^{-1} t B=t h$, then $B^{-\beta} C B^{\beta}=C^{2} t^{\beta} h^{1 / \beta(\beta-1)}$. If $\beta$ is the order of the operator of $G^{\prime}$ that corresponds to $B$, then $t^{\beta} h^{3 / 3 \beta(\beta-1)}=1$, and if the order of $G$ (and hence $\beta$ ), is odd, then $t^{\beta}=1$. We have therefore proved the following:

Theorem I.-If $G$ is a group of the third class, of odd order, the order of every commutator of $G$ is the order of some operator of $G^{\prime}$.

If the order of $G$ is even, the order of any commutator is at most twice the order of some operator of $G^{\prime}$.

Let $G$ be a group of the third class, of odd order, and let $A_{i}(i=1,2, \ldots, n)$ be operators of $G$ that correspond respectively to the generators $A_{i}^{\prime}$ of $G^{\prime}$. Also let $A_{1}^{-1} A_{j} A_{1}=A_{j} t_{j}, A_{1}^{-1} t_{j} A_{1}=t_{j} h_{j}$, and let $\beta_{j}$ be the order of $t_{j}$. Then $A_{1}^{-\beta_{j}} A_{j} A_{1}^{\beta_{j}}=A_{j}$. Therefore if $\mu$ is the least common multiple of the $\beta_{j}(j=2,3, \cdots, n), A_{1}^{\mu}$ is commutative with every operator of $G$; moreover, $A_{1}^{\mu^{\prime}}$, where $\mu^{\prime}<\mu$, is not commutative with every operator of $G$. We have therefore

Theorem II.-If $G$ is a group of the third class, of odd order, the order of every operator of $G^{\prime}$ is the order of a commutator of $G$.

In proving this theorem we assumed that in a group of the third class, of odd order, the least common multiple of the orders of any commutators is the order of a commutator. This is evidently the case in any group whose order is a power of a prime. Suppose now that $G$ is a group of the third class of order $p_{1}^{a_{1}} p_{2}^{a_{2}} \cdots p_{n}^{a_{n}}$, where $p_{1}, p_{2}, \cdots, p_{n}$ are distinct odd primes. It is the direct product of groups $G_{1}, G_{2}, \ldots, G_{n}$ of orders $p_{1}^{a_{1}}, p_{2}^{a_{2}}, \ldots, p_{n}^{a_{n}}$ respectively, and $G^{\prime}$ is the direct product of $G_{1}^{\prime}, G_{2}^{\prime}, \ldots, G_{n}^{\prime}$.*

If $G^{\prime}$ has an operator of order $p_{1}^{a_{1}^{\prime}} p_{2}^{a_{2}^{\prime}} \ldots p_{n}^{a_{n}^{\prime}}$, then $G_{i}^{\prime}$ has an operator of order $p_{i}^{i_{i}^{\prime}}(i=1,2, \ldots, n)$. Therefore $G_{i}$ contains a commutator of order $p_{i}^{a_{i}^{\prime}}$. If we take such a commutator from each of the $n$ factors groups and form their product, we shall get a commutator of $G$ of order $p_{1}^{a_{1}^{\prime}} p_{2}^{a_{2}^{\prime}} \cdots p_{n}^{a_{n}^{\prime}}$.

If the order of $G$ is even, the order of any operator of $G^{\prime}$ is either the order of some commutator of $G$, or twice the order of some commutator of $G$.

In general, if $G$ is of the $n$th class, the least common multiple of the orders of any of its operators is the order of some operator. $\dagger$

Theorem III.-A metabelian group $G^{\prime}$, of odd order, having a set of generators such that the order of one of them is not a divisor of the least common multiple of the orders of all the others, cannot be a group of cogredient isomorphisms.

* Miller, Bulletin of the American Mathematical Society, vol. 5 (1899), p. 294.

†The proof of this is similar to that given by Frobenius and STICKELBerger, Crelle's Journal, vol. 86 (1879), p. 234, for operators of an abelian group.

Trans. Am. Math. Soc. 23. 
Let $A_{i}(i=1,2, \cdots, n)$ be operators of $G$ that correspond respectively to the generators $A_{i}^{\prime}$ of $G^{\prime}$. Let $\alpha_{i}$ be the order of $A_{i}^{\prime}$. Then

$$
A_{1}^{-a_{i}} A_{i} A_{1}^{a_{i}}=A_{i} \quad(i=2,3, \cdots, n) .
$$

If $\mu$ is the least common multiple of $\alpha_{2}, \alpha_{3}, \cdots, \alpha_{n}$, we have

$$
A_{1}^{-\mu} A_{i} A_{1}^{\mu}=A_{i} \text {. }
$$

Therefore $A_{1}^{\mu}$ is invariant in $G$, and $\alpha_{i}$ is a divisor of $\mu$.

CoRolitary.-If $G^{\prime}$ is of order $p^{a}$, where $p$ is an odd prime, it must have at least two generators of the highest order.

That the theorem does not hold for groups of even order is seen in the groups of order 16 of the third class. The group of cogredient isomorphisms of such a group is the non-quaternion group of order 8 . This group is generated by an operator of order 4 and one of order 2. The more general statement of the theorem is as follows:

$A$ metabelian group $G^{\prime}$ having a set of generators such that the order of any one of them is not a divisor of twice the least common multiple of the orders of all the others cannot be a group of cogredient isomorphisms.

If a group $G^{\prime}$ has a set of generators such that some operator $A^{\prime}$ (other than identity) of $G^{\prime}$ is a power of every one of these generators, $G^{\prime}$ cannot be a group of cogredient isomorphisms. For, if it could, the operators of $G$ that correspond to $A^{\prime}$ would be invariant in $G$. In particular, no Hamiltonian group can be a group of cogredient isomorphisms. *

Hence if $G$ is of the third class, of odd order, and has a cyclic commutator subgroup, $G^{\prime}$ must have invariant operators besides its commutators. For if it had no invariant operators besides its commutators, one of these commutators would be a power of every generator of $G^{\prime}$. It follows from this that a group of order $p^{4}$ cannot have a cyclic commutator subgroup of order $p^{2}$ if $p$ is an odd prime.

THeORÈ IV.-If the commutator subgroup of a group $G$ of order $p^{a}$ is of order $p^{\beta}$, where $p$ is a prime, the class of $G$ is equal to, or less than, $\beta+1$.

For if $G$ is non-abelian and all its commutators are invariant, it is of the second class. If only $1 / p$ of the operators of the commutator subgroup are invariant, $G$ is of the third class. Therefore, if the commutator subgroup of $G$ is of order $p^{2}, G$ belongs to the second or third class. If now $G^{\prime}$ belongs to the class $j$, where $j \equiv i$, its commutator subgroup being of order $p^{i-1}$, it follows that the class of any group having $G^{\prime}$ for its group of cogredient isomorphisms is equal to, or less than, $i+1$, and that the order of its commutator

* Miller, Bulletin of the American Mathematical Society, vol. 6 (1900), p. 339. 
subgroup is equal to, or greater than, $p^{i}$. It has just been shown that this supposition holds when $i=3$. Therefore it holds for all values of $i$.

Theorem V.-If $G$ is a group of the third class of order $p^{a}$, where $p$ is a prime, and $G^{\prime}$ has a cyclic commutator subgroup, then the group of cogredient isomorphisms $G^{\prime \prime}$ of $G^{\prime}$ has just two independent generators.*

Since $G^{\prime}$ is metabelian and has a cyclic commutator subgroup, $G^{\prime \prime}$ must have an even number of independent generators. We shall show that there cannot be four of these generators and an obvious extension of the same argument will show that there cannot be any even number of them greater than two.

Let the four independent generators of $G^{\prime \prime}$ be $A^{\prime \prime}, B^{\prime \prime}, C^{\prime \prime}, D^{\prime \prime}$; and let $A^{\prime}$, $B^{\prime}, C^{\prime}, D^{\prime}$ be operators of $G^{\prime}$ that correspond respectively to these, while operators of $G$ that correspond to $A^{\prime}, B^{\prime}, C^{\prime}, D^{\prime}$ are represented by $A, B, C$, $D$ respectively. Let $h_{i}(i=1,2, \cdots)$ represent the invariant operators of $G$, $t$ a commutator of $G$ that corresponds to a generator of the cyclic commutator subgroup of $G^{\prime}$, and $k_{j}(j=1,2, \cdots)$ operators of $G$ that correspond to the invariant operators of $G^{\prime}$ that are not commutators (if there be such operators). Let $p^{m}$ be the order of the commutator subgroup of $G^{\prime}$. Two of the independent generators of $G^{\prime \prime}$, say $C^{\prime \prime}$ and $D^{\prime \prime}$, will then be of order $p^{m}$, and the other two, $A^{\prime \prime}$ and $B^{\prime \prime}$, will be of order $p^{m-\beta}$, where $0 \equiv \beta<m$. Now, the commutator of $G^{\prime}$ formed by $A^{\prime}$ and $B^{\prime}$ is of order $p^{m-\beta}$, and that formed by $C^{\prime}$ and $D^{\prime}$ is of order $p^{m}$. Every operator of $G^{\prime}$ that corresponds to the subgroup of $G^{\prime \prime}$ generated by $C^{\prime \prime}$ and $D^{\prime \prime}$ is commutative with $A^{\prime}$ and $B^{\prime}$; likewise every operator of $G^{\prime}$ that corresponds to the subgroup of $G^{\prime \prime}$ generated by $A^{\prime \prime}$ and $B^{\prime \prime}$ is commutative with $C^{\prime}$ and $D^{\prime}$. Therefore

Hence

$$
D^{-1} B D=B h_{1}, \quad D^{-1} C D=C t h_{2}, \quad C^{-1} B C=B h_{3} .
$$

$$
\left(C t h_{2}\right)^{-1} B h_{1}\left(C t h_{2}\right)=B h_{1} h_{3},
$$

and $t$ is commutative with $B$. It can be shown in a similar way that $t$ is commutative with $k_{j}$ and $A$; also that $t^{p^{\beta}}$ is commutative with $C$, and $D$. Therefore $t^{\beta}$ is invariant in $G$. But this cannot be the case since $\beta<m$. The supposition that $G^{\prime \prime}$ has four independent generators leads therefore to a contradiction.

TheOREM VI.-In any group $G$ the operators that correspond to the invariant operators of $G^{\prime}$ are commutative with all the commutators of $G$.

Let $t$ be any commutator of $G$ and let $A_{1}$ and $A_{2}$ be so chosen that

$$
A_{2}^{-1} A_{1} A_{2}=A_{1} t \text {. }
$$

If $k$ is any operator of $G$ that corresponds to an invariant operator of $G^{\prime}$, then

\footnotetext{
* For a special case of this theorem, see Young, loc. cit., p. 178.
} 


$$
k^{-1} A_{1} k=A_{1} h_{1}, \quad k^{-1} A_{2} k=A_{2} h_{2},
$$

where $h_{1}$ and $h_{2}$ are invariant operators of $G$.

Therefore

$$
\left(k h_{2}^{-\mathrm{i}}\right)^{-1} A_{1} t\left(k h_{2}^{-1}\right)=A_{1} t h_{1},
$$

and $k$ is commutative with $t$.

We shall now prove that there is one, and only one, group $G$ of order $p^{5}$, where $p$ is an odd prime, that has a cyclic commutator subgroup of order $p^{2}$.

Such a group could not be abelian or metabelian. It must therefore be of the third class. Also $G^{\prime}$ must be of order $p^{4}$ of the type $(1,1)(1,1)$. It cannot have one generator of order greater than the orders of all the others, and it must contain an operator of order $p^{2}$, since $G$ contains a commutator of order $p^{2}$. There is only one metabelian group of order $p^{4}$ that satisfies these conditions.* It is generated by the operators $A^{\prime}$ and $B^{\prime}$, where

$$
A^{\prime p^{2}}=1, \quad B^{\prime p^{2}}=1, \quad B^{\prime-1} A^{\prime} B^{\prime}=A^{\prime 1+p} .
$$

Let $t$ be a commutator of $G$ of order $p^{2}$, and let $A$ and $B$ be operators of $G$ that correspond respectively to $A^{\prime}$ and $B^{\prime}$ of $G^{\prime}$. We may assume that $A^{\prime}$ has been so chosen that

$$
A^{p}=t, \quad B^{p^{2}}=t^{b p}, \quad B^{-1} A B=\cdot A^{1+a p}, \quad a \neq 0(\bmod . p) .
$$

If $b=0$, these relations define a group. If $0<b<p$, let $B_{1} \equiv B A^{-b}$. Then $B_{1}^{p^{2}}=\left(B A^{-b}\right)^{p^{2}}=B^{p^{2}} A^{-b p^{2}}=1$. The group $\{A, B\}$ is identical with the group $\left\{A, B_{1}\right\}$. This is the same as the one obtained when $b=0$.

This group is not found in the published list of groups of order $p^{5} \cdot \dagger$ This list contains two groups of order $3^{5}$ and one of order $p^{5}(p>3)$, whose commutator subgroups are cyclic of order $p^{2}$. It follows from Theorem III that these three groups do not exist.

Suppose that $G$ is a group of order $p^{6}$ that has a cyclic commutator subgroup of order $p^{3}, p$ being an odd prime. Such a group must be of the fourth class and its group of cogredient isomorphisms must be of order $p^{5}$ and must have a cyclic commutator subgroup of order $p^{2}$. We have just seen that there is only one such group. But it cannot be a group of cogredient isomorphisms since it is generated by an operator of order $p^{3}$ and one of order $p^{2}$. Hence, there is no group of order $p^{6}$ that has a cyclic commutator subgroup of order $p^{3}, p$ being an odd prime. 410.

* Young, loc. cit., p. 147 ; HöLder, Mathematische $\Lambda$ nnalen, vol. 43 (1893), pp. 409,

† BAGNers, A n nali di Matematica, series III, vol. 1 (1898), pp. 137-228. It is given by BuRnside, Theory of Groups of Finite Order, 1897, p. 79. 
If $G$ is a group of the fourth class whose order is not divisible by 2 or 3, and if $A_{1}$ and $A_{2}$ are any two operators of $G$ then

and

$$
A_{2}^{-1} A_{1} A_{2}=A_{1} t_{1}
$$

where

$$
A_{2}^{-a_{1}} A_{1} A_{2}^{a_{1}}=A_{1} t_{1}^{a_{1}} t_{2}^{\frac{a_{1}\left(a_{1}-1\right)}{2}} h_{1}^{\frac{a_{1}\left(a_{1}-1\right)\left(a_{1}-2\right)}{2 \cdot 3}} h_{2}^{\frac{a_{1}\left(a_{1}-1\right)\left(a_{1}-2\right)}{2 \cdot 3}},
$$

$$
A_{2}^{-1} t_{1} A_{2}=t_{1} t_{2}, \quad A_{2}^{-1} t_{2} A_{2}=t_{2} h_{1}, \quad t_{1}^{-1} t_{2} t_{1}=t_{2} h_{2},
$$

$h_{1}$ and $h_{2}$ being invariant operators of $G$.

If $G$ is of the fifth class, we have

$$
A_{2}^{-a_{1}} A_{1} A_{2}^{a_{1}}=A_{1} t_{1}^{a_{1}} t_{2}^{\frac{a_{1}\left(a_{1}-1\right)}{2}} t_{3}^{\frac{a_{1}\left(a_{1}-1\right)\left(a_{1}-2\right)}{2 \cdot 3}} h_{1}^{\frac{a_{1}\left(a_{1}-1\right)\left(a_{1}-2\right)}{2 \cdot 3}} h_{2}^{\frac{a_{1}\left(a_{1}-1\right)\left(a_{1}-2\right)\left(a_{1}-3\right)}{2 \cdot 3 \cdot 4}},
$$

where

$$
A_{2}^{-1} t_{2} A_{2}=t_{2} t_{3}, \quad t_{1}^{-1} t_{2} t_{1}=t_{2} h_{1}, \quad A_{2}^{-1} t_{3} A_{2}=t_{3} h_{2} .
$$

Now, if $\alpha_{1}$ is the order of the operator of $G^{\prime}$ that corresponds to $A_{1}$, then the orders of $t_{1}, t_{2}, t_{3}, h_{1}, h_{2}$ are all divisors of $\alpha_{1}$. Hence in a group $G$ of the fourth or fifth class whose order is not divisible by 2 or 3 , an operator $A_{1}$ that corresponds to an operator of $G^{\prime}$ of order $\alpha_{1}$ is commutative with the $\alpha_{1}$ power of every operator of $G$.

From this follows

Theorem VII.-A group $G^{\prime}$ of the third or fourth class whose order is not divisible by 2 or 3 cannot be a group of cogredient isomorphisms if it has a set of generators such that the order of one of them is not a divisor of the least common multiple of the orders of all the others.

Cornell University, June, 1901. 